\title{
Phrase truncation in PubMed searches
}

Roberta Shanman, MLS

See end of article for author's affiliation.

Comment on Duffy S, de Kock S, Misso K, Noake C, Ross J, Stirk L. Supplementary searches of PubMed to improve currency of MEDLINE and MEDLINE In-Process searches via Ovid. J Med Libr Assoc. 2016 Oct;104(4):309-12. DOI: http://dx.doi.org/10.3163/1536-5050.104.4.011.

To the editor, in their "Methods" section, the authors of "Supplementary Searches of PubMed to Improve Currency of MEDLINE and MEDLINE InProcess Searches via Ovid" state that "PubMed does not accept truncation when phrase searching, so we had to use numerous phrases to capture as many variations as possible."

In fact, if an asterisk is added to the end of a phrase, truncation is performed. No double quotes are required around the phrase, as the asterisk acts as a phrase marker as well as truncation. The PubMed searches in the supplementary material duplicate the search string used in the article. Sets 3 and 9 utilize the phrase truncation feature (figure 1). The number of results is the same for the terms using this feature as it is for each individual variation of the term using a Boolean "OR."

\section{AUTHOR'S AFFILIATION}

Roberta Shanman, MLS, roberta@rand.org, Reference and Consulting Research Librarian, RAND Corporation, Santa Monica, CA

Received May 2017; accepted June 2017

Figure 1 Sample truncation in PubMed

\begin{tabular}{|c|c|c|c|}
\hline$\# 10$ & $\underline{\text { Add }}$ & Search (\#5 OR \#6) & $\underline{92815}$ \\
\hline$\rightarrow \underline{\# 9}$ & $\underline{\text { Add }}$ & Search prostate cancer*[tiab] & $\underline{92815}$ \\
\hline$\# 8$ & $\underline{\text { Add }}$ & Search "prostate cancerous"[tiab] Schema: all & $\underline{0}$ \\
\hline$\# 7$ & $\underline{\text { Add }}$ & Search "prostate cancerous"[tiab] & $\underline{0}$ \\
\hline$\# 6$ & $\underline{\text { Add }}$ & Search "prostate cancers"[tiab] & $\underline{5002}$ \\
\hline$\# \underline{F}$ & $\underline{\text { Add }}$ & Search "prostate cancer"[tiab] & $\underline{91372}$ \\
\hline$\# 4$ & $\underline{\text { Add }}$ & Search (\#1 OR \#2) & $\underline{6800}$ \\
\hline$\nabla \underline{\# 3}$ & $\underline{\text { Add }}$ & Search prostate carcinoma*[tiab] & $\underline{6800}$ \\
\hline$\# 2$ & $\underline{\text { Add }}$ & Search "prostate carcinomas"[tiab] & $\underline{974}$ \\
\hline$\# 1$ & $\underline{\text { Add }}$ & Search "prostate carcinoma"[tiab] & 6127 \\
\hline
\end{tabular}

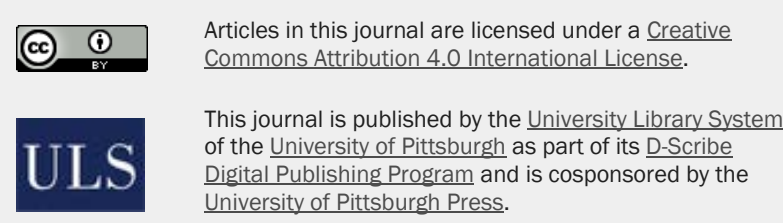

ISSN 1558-9439 (Online) 